\title{
Poly-pharmacy among the elderly in a Danish emergency department
}

\author{
Camilla Kobylecki, Thomas Andersen Schmidt \\ From 4th Danish Emergency Medicine Conference \\ Roskilde, Denmark. 25-26 November 2011
}

\section{Background}

A substantial part of the clientele in an emergency department setting are elderly patients who often consume several prescription medications daily hereby increasing the risk of potential drug-drug interactions. We examined the extent of poly-pharmacy at the time of admission among a group of elderly patients.

\section{Methods}

All patients in the age range 65-79 years admitted consecutively to Holbaek Emergency Department during one week were included. Information on medication at the time of admission was obtained from medical charts.

\section{Results}

A total of 73 patients, $48 \%$ women and $52 \%$ men were included, the average age being 72 years. The most frequent reasons for admission were lung diseases (21\%), neurological disease (25\%), cardiac diseases (15\%) and infections (12\%). The patients used on average 6.6 prescription medications daily, ranging from 0 to 18 different drugs. 10 patients received 1 drug or less. The most frequently used drugs in this group were anti-hypertensive medication (56\% of the patients), blood-cholesterol lowering drugs (55\% of the patients), drugs to prevent thrombosis (56\% of the patients), pain medication (37\% of the patients) and diuretics (36\% of the patients).

A total of 41 patients were prescribed anti-hypertensive medications. 20 patients were prescribed more than one drug to control blood pressure and 5 of these patients were treated with more than two different drugs to control blood pressure. 18 patients in antihypertensive treatment were also treated with diuretics. The most frequently prescribed anti-hypertensive drugs were calcium antagonists $(n=15)$, beta-receptor blockers $(\mathrm{n}=15)$ and ACE-inhibitors $(\mathrm{n}=14)$.

A total of 40 patients were prescribed drugs to lower blood cholesterol and $75 \%(n=30)$ of these were treated with simvastatin. 41 patients were prescribed drugs to prevent thrombosis, among these acetylsalicylic acid $(n=25)$, clopidogrel $(n=11)$ and warfarin $(n=10)$ were the most frequently used drugs.

27 patients were treated with pain medication at the time of admission and 15 of these patients received opioids.

\section{Conclusion}

Cardiovascular drugs are the most predominantly prescribed drugs for elderly patients. Poly-pharmacy is in particular seen among antihypertensive medications. Caution in prescribing new drugs due to potential drugdrug interactions poses a challenge for the attending physician.

Published: 16 April 2012

doi:10.1186/1757-7241-20-S2-P39

Cite this article as: Kobylecki and Schmidt: Poly-pharmacy among the elderly in a Danish emergency department. Scandinavian Journal of Trauma, Resuscitation and Emergency Medicine 2012 20(Suppl 2):P39.

* Correspondence: camilla.kobylecki@gmail.com

The Emergency Department, Holbæk University Hospital, Denmark 